\title{
Remarks on the mean-square values of the geomagnetic field and its components
}

\author{
Angelo De Santis $\left({ }^{1}\right)$, Claudia Falcone $\left({ }^{1}\right)$ and Frank J. Lowes $\left({ }^{2}\right)$ \\ (') Istituto Nazionale di Geofisica, Roma, Italy \\ $\left.{ }^{2}\right)$ Physics Department, University of Newcastle, Upon Tyne, U.K.
}

\begin{abstract}
When considering functions on the Earth's (spherical) surface, mean-square values are often used to indicate their (relative) magnitude. If a function is separated into its (essentially) spherical harmonic components then, provided these individual harmonic components are orthogonal over the surface, the concept of spatial power spectrum can be introduced, with each harmonic contributing separately to the total mean square value; this is true for the geomagnetic field vector $\boldsymbol{B}$, its horizontal component vector $\boldsymbol{H}$, and its vertical component $Z$. However, because of the lack of orthogonality this concept is not applicable to the horizontal $X$ and $Y$ components individually; problems which arise from this are discussed.
\end{abstract}

Key words spherical harmonics - orthogonality geomagnetic spectrum

\section{Introduction}

In current-free regions the scalar potential $V$ of the internal geomagnetic field $\boldsymbol{B}$ can be expanded in spherical harmonics:

$$
\begin{aligned}
V(r, \theta, \lambda) & =a \cdot \sum_{n=1}^{\infty}\left(\frac{a}{r}\right)^{n+1} \sum_{m=0}^{n}\left[g_{n}^{m} \cos m \lambda+\right. \\
& \left.+h_{n}^{m} \sin m \lambda\right] P_{n}^{m}(\cos \theta),
\end{aligned}
$$

where $\theta, \lambda$ are geocentric colatitude and longitude, $r$ is the distance from the centre of the Earth, $a=6371.2 \mathrm{~km}$ is the Earth's mean radius, $P_{n}^{m}$ are the Schmidt semi-normalized associated Legendre polynomials, $g_{n}^{m}, h_{n}^{m}$ are the Gauss coefficients; $n, m$ are the degree and order of the spherical harmonic expansion. In practice, the degree $n$ is truncated at a maximum value $N$, after which the contribution of the harmonics with higher degree is considered negligible.

The expression (1.1) for the magnetic potential arises in the physical context as a solution of Laplace's equation.

To enable us to use compact expressions we will ignore terms involving $h_{n}^{m}$ (except where the sum over $m$ is made explicit), so at $r=a$ (a good approximation to the Earth's surface) we can write

$$
\begin{aligned}
V(a, \theta, \lambda) & =a \cdot \sum_{n, m} g_{n}^{m} \cos m \lambda P_{n}^{m}(\cos \theta)= \\
& =a \cdot \sum_{n, m} g_{n}^{m} \Psi_{n}^{m}(\theta, \lambda)
\end{aligned}
$$

where the $\Psi_{n}^{m}$ are surface harmonics.

These surface harmonics form the solution of the angular part of Laplace's equation so, from Sturm Liouville theory (e.g. Arfken, 
1985), they form a complete orthogonal set over the sphere; this important property is expressed by the following relation (e.g. Chapman and Bartels, 1940):

$$
\begin{aligned}
& \int_{4 \pi} \Psi_{n}^{m} \Psi_{n^{\prime}}^{m^{\prime}} d \Omega=\int_{0}^{2 \pi} \cos m \lambda \cos m^{\prime} \lambda d \lambda \\
& \times \int_{-1}^{1} P_{n}^{m}(x) P_{n^{\prime}}^{m}(x) d x=\frac{4 \pi}{2 n+1} \delta_{m, m^{\prime}} \delta_{n, n^{\prime}}
\end{aligned}
$$

where $\delta_{i, j}$ is the Kronecker delta, and $\Omega$ is the solid angle at the centre, such that $d \Omega=d \lambda d(\cos \theta)=d \lambda d x$, where $x=\cos \theta$. In particular, eq. (1.3) is an orthogonal relation of the kind

$$
\int_{S} f \cdot g d \Omega=\|f\|^{2} \delta_{f, g}
$$

where this integral is the scalar product between functions $f, g$ integrated over the space $S$ and $\|f\|$ is the Euclidean norm of $f$.

Mathematically, eq. (1.3) derives from the reciprocal orthogonality of Fourier functions $\cos m \lambda, \cos m^{\prime} \lambda$ over the interval $[0,2 \pi]$, and that of associated Legendre Polynomials $P_{n}^{m}(x), P_{n^{\prime}}^{m}(x)$ over $[-1,1]$ :

$$
\begin{aligned}
& \int_{0}^{2 \pi} \cos m \lambda \cos m^{\prime} \lambda d \lambda=\pi \delta_{m, m^{\prime}}\left(1+\delta_{0, m}\right) \\
& \int_{-1}^{1} P_{n}^{m}(x) P_{n^{\prime}}^{m}(x) d x=\frac{2}{2 n+1} \delta_{n, n^{\prime}}\left(2-\delta_{0, m}\right) .
\end{aligned}
$$

If values of the potential $V$ were known on a sphere, then for a sufficiently uniform distribution, the orthogonality of the spherical harmonics permits the use of either least-squares or integration methods to estimate the values of the Gauss coefficients. We will see more details in section 4.

The property of orthogonality suggests that the surface spherical harmonics constitute the natural spectral representation for the potential and any other function that vary over the sphere. In this way «spherical harmonics are a device for studying variations over the spherical surface analogous to Fourier series for vari- ation in time» (Kaula, 1967). Therefore, in a way similar to Fourier analysis, it is possible to introduce the concept of a (spatial) power spectrum of the potential, of the magnetic field and, if possible, of its components.

In practice the potential is not a direct observable, and we have to determine the Gauss coefficients from the geomagnetic field $\boldsymbol{B}=-\nabla V$, or its components based on a local coordinate system:

$$
(X, Y, Z)=\left(\frac{\partial}{r \partial \theta},-\frac{\partial}{r \sin \theta \partial \lambda}, \frac{\partial}{\partial r}\right) V .
$$

We can expand the magnetic potential

$$
V=\sum_{n, m} g_{n}^{m} V_{n}^{m},
$$

with the obvious definition of $V_{n}^{m}$. Consequently the magnetic field $\boldsymbol{B}$ can be expanded as

$$
\boldsymbol{B}=\sum_{n, m} g_{n}^{m} \boldsymbol{B}_{n}^{m}, \quad \boldsymbol{B}_{n}^{m}=-\nabla V_{n}^{m} .
$$

Analogously we can write:

$$
(X, Y, Z)=\sum_{m, n} g_{n}^{m}\left(X_{n}^{m}, Y_{n}^{m}, Z_{n}^{m}\right)
$$

where we introduce the decomposition into spectral elements $X_{n}^{m}, Y_{n}^{m}, Z_{n}^{m}$ defined as:

$$
\begin{gathered}
X_{n}^{m}=\left(\frac{a}{r}\right)^{n+2} \frac{\partial}{\partial \theta} \Psi_{n}^{m}(\theta, \lambda)= \\
=\left(\frac{a}{r}\right)^{n+2} \cos m \lambda \frac{d}{d \theta} P_{n}^{m}(\cos \theta), \\
Y_{n}^{m}=-\left(\frac{a}{r}\right)^{n+2} \frac{1}{\sin \theta} \frac{\partial \Psi_{n}^{m}(\theta, \lambda)}{\partial \lambda}= \\
=m\left(\frac{a}{r}\right)^{n+2} \sin m \lambda \frac{P_{n}^{m}(\cos \theta)}{\sin \theta}, \\
Z_{n}^{m}=-(n+1)(a / r)^{n+2} \Psi_{n}^{m}(\theta, \lambda)= \\
=-(n+1)(a / r)^{n+2} \cos m \lambda P_{n}^{m}(\cos \theta) .
\end{gathered}
$$


These expressions are not themselves all orthogonal: in particular those for $X_{n}^{m}$ (i.e. $\partial \Psi_{n}^{m} / \partial \theta$; this fact does not explicitly emerge from sect. 17.14 of Chapman and Bartels, 1940), and for $Y_{n}^{m}$ (which are of the form $\Psi_{n}^{m} / \sin \theta$ ). We will see in the next section the consequences of this when deriving $X$ and $Y$ components, and their mean-square values, from the magnetic potential $V$.

In literature, these same spectral elements are also denoted as $\partial / \partial g_{n}^{m}(X, Y, Z)(e . g$. Langel, 1987). Sometimes they include only their $\theta$-dependence (e.g. Schmidt, 1935; Chakrabarty, 1954).

For a particular pair $(n, m)$ the relative value of $g_{n}^{m}$ and $h_{n}^{m}$ depends on the choice of the $\lambda=0$ origin, but the sum $\left[\left(g_{n}^{m}\right)^{2}+\left(h_{n}^{m}\right)^{2}\right]$ is invariant to the choice of this origin. For a given $n$ all the coefficients change if the $\theta=0$ axis is changed, but the sum $\sum_{m}\left[\left(g_{n}^{m}\right)^{2}+\left(h_{n}^{m}\right)^{2}\right]$ is invariant. We will see in the next section that it is this sum which is relevant to the meansquare values.

In many papers the mean-square values over the sphere $r=a$ of functions such as $V, \boldsymbol{B}, \boldsymbol{H}$, $X, Y, Z$ are determined, often with the aim of resolving the total mean-square value into a series of parts, each of which depends only on the corresponding degree $n$, or spatial frequency, so giving a (one-dimensional) spatial power spectrum. This resolution is certainly possible for $V, \boldsymbol{B}, \boldsymbol{H}$ and $Z$, but it is not for $X$ and $Y$.

Physically, the difference between $V, \boldsymbol{B}, \boldsymbol{H}$, $Z$ and $X, Y$, is that the former group have values, and hence mean-squares, which are invariant with respect to the choice of axis $\theta=0$, while the latter are not. Mathematically the difference is that if the former are expanded in terms of surface harmonics, the individual $V_{n}^{m}$ etc. are orthogonal, while for the latter they are not.

This paper discusses some of the problems resulting from this difference. Even though much of this work is based on already published material (especially the fundamental works of Chapman and Bartels (1940), Mauersberger (1956, 1959), Lucke (1957, 1959) and Lowes $(1966,1974)$ ), it tries to give a simple and organic view of the matter to clarify some concepts that could be otherwise misinterpreted or disregarded. Section 2 derives expressions for the mean value (over the sphere) of products of the different spectral elements and total mean-square values, section 3 applies these results to the power spectra, and section 4 will recall some general results on integral and least-squares methods, and the possible use of $X \sin \theta$ and $Y \sin \theta$; section 5 completes the work with a general discussion.

\section{Scalar products of spectral components of $\boldsymbol{B}, \boldsymbol{H}$ and $X, Y, Z$}

When writing eqs. (1.2) and (1.5) we introduced a spectral decomposition for $V$ and $\boldsymbol{B}$ : our problem is that of computing the scalar products of their spectral elements or, which is the same (apart from a proportional factor), the mean value over the sphere of their products and, as a special case, their mean-square values.

Let us denote $\langle\omega\rangle$ as the mean value of a function $\omega(r, \theta, \lambda)$ on the surface $r=\mathrm{a}$ :

$$
<\omega>=\frac{1}{4 \pi} \int_{4 \pi} \omega(a, \theta, \lambda) d \Omega .
$$

The mean value of the product between two functions $f(a, \theta, \lambda), g(a, \theta, \lambda)$ is directly related to their scalar product:

$$
<f g>=\frac{1}{4 \pi} \int_{4 \pi} f(a, \theta, \lambda) g(a, \theta, \lambda) d \Omega,
$$

with the obvious generalization if $\boldsymbol{f}$ and $\boldsymbol{g}$ are vectors. $<f^{2}>$.

If $f=g$, we have the mean-square value

We are now going to discuss whether the spectral components introduced in expressions (1.5) are orthogonal or not, i.e. whether $\langle f g\rangle$ is zero when $f$ and $g$ are different spectral-components.

The expression for $\left\langle V^{2}\right\rangle$ comes directly 
from the orthogonality of spherical harmonics (eqs. (1.2), (1.3)),

$$
\begin{aligned}
<V^{2}> & =<\sum_{n, n^{\prime}, m, m^{\prime}} g_{n}^{m} g_{n^{\prime}}^{m^{\prime}} V_{n}^{m} V_{n^{\prime}}^{m^{\prime}}>= \\
& =a^{2} \sum_{n, m}\left(g_{n}^{m}\right)^{2}<\left(\Psi_{n}^{m}\right)^{2}>,
\end{aligned}
$$

which can be expanded as

$<V^{2}>=\sum_{n=1}^{N} \frac{a^{2}}{2 n+1} \sum_{m=0}^{n}\left[\left(g_{n}^{m}\right)^{2}+\left(h_{n}^{m}\right)^{2}\right] ;$

note that the value of the sum over $m$ is independent of the choice of axis.

Just as the potentials $V_{n}^{m}(r, \theta, \lambda)$ are orthogonal over the sphere, so also are the corresponding $\boldsymbol{B}_{n}^{m}=-\nabla V_{n}^{m}$. If we define the field magnitude $F=|\boldsymbol{B}|$, then because of the orthogonality we have

$$
\begin{aligned}
\left.<F^{2}\right\rangle & =\langle\boldsymbol{B} \cdot \boldsymbol{B}\rangle=\sum_{n, m}\left(g_{n}^{m}\right)^{2}\left\langle\boldsymbol{B}_{n}^{m} \cdot \boldsymbol{B}_{n}^{m}\right\rangle= \\
& =\sum_{n=1}^{N}(n+1) \sum_{m=0}^{n}\left[\left(g_{n}^{m}\right)^{2}+\left(h_{n}^{m}\right)^{2}\right] .
\end{aligned}
$$

The first general proof we can find of this is in Stratton, 1941 (page 417), with the first proof in a geophysical context by Jeffries (1942).

Although it is not explicitly stated, Stratton (1941) also includes the result that the surface (horizontal) vector components $\boldsymbol{H}_{n}^{m}=\left(X_{n}^{m}, Y_{n}^{m}\right)$ are also orthogonal over the sphere, giving

$$
\begin{aligned}
& \left.<\boldsymbol{H} \cdot \boldsymbol{H}>=\sum_{n, m}\left(g_{n}^{m}\right)^{2}<\boldsymbol{H}_{n}^{m} \cdot \boldsymbol{H}_{n}^{m}\right\rangle= \\
& =\sum_{n=1}^{N} \frac{n(n+1)}{2 n+1} \sum_{m=0}^{n}\left[\left(g_{n}^{m}\right)^{2}+\left(h_{n}^{m}\right)^{2}\right] .
\end{aligned}
$$

It is obvious from their definition (1.5), and also follows from (2.2) and (2.3), that the vertical components $Z_{n}^{m}$ are also orthogonal, giving

$$
<Z^{2}>=\sum_{n, m}\left(g_{n}^{m}\right)^{2}\left(Z_{n}^{m}\right)^{2}=
$$

$$
=\sum_{n=1}^{N} \frac{(n+1)^{2}}{2 n+1} \sum_{m=0}^{n}\left[\left(g_{n}^{m}\right)^{2}+\left(h_{n}^{m}\right)^{2}\right]
$$

These results were given in the geomagnetic context by Lucke (quoted by Mauersberger, 1956) and independently by Lowes (1966).

But if we express $\boldsymbol{B}$ as $(X, Y, Z)$ we obtain

$$
\begin{gathered}
<F^{2}>=<X^{2}>+<Y^{2}>+<Z^{2}>= \\
=\sum_{n, n^{\prime}, m, m^{\prime}} g_{n}^{m} g_{n^{\prime}}^{m^{\prime}}\left(<X_{n}^{m} X_{n^{\prime}}^{m^{\prime}}>+<Y_{n}^{m} Y_{n^{\prime}}^{m^{\prime}}>+<Z_{n}^{m} Z_{n^{\prime}}^{m^{\prime}}>\right),
\end{gathered}
$$

and, as we now show, the $X_{n}^{m}$ are not all orthogonal over the sphere, nor are the $Y_{n}^{m}$. From eq. (1.5) we have for $X$ :

$$
\begin{gathered}
<X_{n}^{m} X_{n^{\prime}}^{m^{\prime}}>=\frac{1}{4} \delta_{m, m^{\prime}}\left[\int_{-1}^{1}\left(1-x^{2}\right) \frac{d P_{n}^{m}}{d x} d P_{n^{\prime}}^{m}\right]= \\
=-\frac{1}{4} \delta_{m, m^{\prime}}\left[\int_{-1}^{1} P_{n^{\prime}}^{m} \frac{d}{d x}\left(\left(1-x^{2}\right) \frac{d P_{n}^{m}}{d x}\right) d x\right]
\end{gathered}
$$

Remembering the associated Legendre equation:

$$
\frac{d}{d x}\left(\left(1-x^{2}\right) \frac{d P_{n}^{m}}{d x}\right)=\frac{m^{2}}{1-x^{2}} P_{n}^{m}-n(n+1) P_{n}^{m}
$$

the right side of (2.6) can be expressed as the sum of two parts, so:

$$
\begin{gathered}
<X_{n}^{m} X_{n^{\prime}}^{m^{\prime}}>= \\
=\delta_{m, m^{\prime}}\left[-\frac{m^{2}}{4} \int_{-1}^{1} \frac{P_{n}^{m} P_{n^{\prime}}^{m}}{1-x^{2}} d x+\frac{n(n+1)}{2 n+1} \delta_{n, n^{\prime}}\right] .
\end{gathered}
$$


The first term in the right side of (2.7) can be called $C_{n, n^{\prime}, m}$ (Lowes, 1975):

$$
C_{n, n^{\prime}, m}=\frac{m^{2}}{4} \int_{-1}^{1} \frac{P_{n}^{m} P_{n^{\prime}}^{m}}{1-x^{2}} d x,
$$

and is non-zero even when $n \neq n^{\prime}$. Similarly, for $Y$ and $Z$ :

$$
\begin{gathered}
<Y_{n}^{m} Y_{n^{\prime}}^{m^{\prime}}>= \\
=\delta_{m, m^{\prime}} \frac{m^{2}}{4} \int_{-1}^{1} \frac{P_{n}^{m} P_{n^{\prime}}^{m}}{1-x^{2}} d x=\delta_{m, m^{\prime}} C_{n, n^{\prime}, m}
\end{gathered}
$$

and

$$
<Z_{n}^{m} Z_{n^{\prime}}^{m^{\prime}}>=\frac{(n+1)^{2}}{2 n+1} \delta_{m, m^{\prime}} \delta_{n, n^{\prime}}
$$

giving (2.4).

Because $C_{n, n^{\prime}, m}$ is present with opposite sign in $X$ and $Y$ (eqs. (2.7) and (2.8)), it does not appear in the expression for $\langle\boldsymbol{H} \cdot \boldsymbol{H}\rangle$ of eq. (2.3), and for $\left\langle F^{2}>\right.$ in eq. (2.2), as it is clear looking at (2.5).

Here we are just interested in the existence of this term, so we will not calculate it (details are given in Lucke, 1957); some idea of its magnitude is given by its value for $n=n^{\prime}$ (Lowes, 1966): $C_{n, n, m}=m / 2$.

We therefore have

$$
\begin{aligned}
<X^{2}> & =\sum_{m, m^{\prime}=0}^{N} \sum_{n=m}^{N} \sum_{n^{\prime}=m^{\prime}}^{N} g_{n}^{m} g_{n^{\prime}}^{m^{\prime}}<X_{n}^{m} X_{n^{\prime}}^{m^{\prime}}>= \\
& =\sum_{n=1}^{N} \sum_{m=0}^{n}\left[\frac{n(n+1)}{2 n+1}-\frac{1}{2} m\right]\left(g_{n}^{m}\right)^{2} \\
& -\sum_{m=0}^{N} \sum_{n=m}^{N} \sum_{n^{\prime}=m}^{n-1} C_{n, n^{\prime}, m} g_{n}^{m} g_{n^{\prime}}^{m}, \\
& <Y^{2}>=\sum_{n=1}^{N} \sum_{m=0}^{n} \frac{1}{2} m\left(g_{n}^{m}\right)^{2}+ \\
& +\sum_{m=0}^{N} \sum_{n=m}^{N} \sum_{n^{\prime}=m}^{n-1} C_{n, n^{\prime}, m} g_{n}^{m} g_{n^{\prime}}^{m},
\end{aligned}
$$

where it is understood that the Gauss coefficients are defined to be zero if any index is less than zero.

Although when $n \neq n^{\prime} C_{n, n^{\prime}, m}$ is less than unity (Lucke, 1957; Lowes, 1975), the second series of sums in (2.10) and (2.11) is not negligible with respect to the first one, giving a significant contribution to $\left\langle X^{2}\right\rangle$ and $\left\langle Y^{2}\right\rangle$.

The scalar products of the form $<X_{n}^{m} X_{n^{\prime}}^{m^{\prime}}>$ contained in expressions (2.7), (2.8) and (2.9) are well known approximations to the elements of the normal equation matrices when data are equally distributed by area over the Earth, or when discussing the weight to give them (Alldredge and Kawasaki, 1981; Schmitz and Cain, 1983; Langel, 1987; Kawasaki et al., 1989); other details will be given in section 4 .

Unfortunatelly Lowes (1966) only gave these results (2.7) and (2.8) for individual harmonics (i.e. for $n=n^{\prime}, m=m^{\prime}$ ), and this has led to some later authors assuming orthogonality of the $X_{n}^{m}, Y_{n}^{m}$. For example Langel (1987, page $355)$ assumes the three components are individually orthogonal (and there are also some typographical errors in his eqs. (122a-c)); in fact his numerical experiments used the full field vector $(X, Y, Z)$, so this did not matter.

\section{Spatial power spectra}

The concept of a spatial power spectrum in geomagnetism was in effect introduced by Lucke $(1957,1959)$ and independently by Lowes (1974). This concept, and its use for the interpretation of the different contributions (crustal and core) was extended by McLeod and Coleman (1980), Langel and Estes (1982) and Cain et al. (1989).

The spectral component $R_{n}(B)$ is «the meansquare value over the sphere of [that part] of $\boldsymbol{B}$ produced by harmonics of degree $n \gg$ (Lowes, 1974). This was possible because on the sphere $r=a$, the degree $n$ specifies the smallest wavelength $2 \pi a / n$ of the corresponding spherical harmonics, and because the $\boldsymbol{B}_{n}^{m}$ were orthogonal. It is evident that this concept can be applied to a field $\boldsymbol{E}$ (or scalar $E$ ) only when the 
$\boldsymbol{E}_{n}^{m}$ are orthogonal; so that

$$
\left.\left.<|\boldsymbol{E}|^{2}\right\rangle=\sum_{m, n}\left(g_{n}^{m}\right)^{2}<\left|\boldsymbol{E}_{n}^{m}\right|^{2}\right\rangle=\sum_{n} R_{n}(\boldsymbol{E}),
$$

where $R_{n}(\boldsymbol{E})$ is defined as follows:

$$
R_{n}(\boldsymbol{E})=\sum_{m}\left[\left(g_{n}^{m}\right)^{2}+\left(h_{n}^{m}\right)^{2}\right]<\left|\boldsymbol{E}_{n}^{m}\right|^{2}>.
$$

It is therefore sensible to apply the concept only to $V, \boldsymbol{B}, \boldsymbol{H}$ and $Z$. The existence of such spectra is possible because the physical variation of these functions over the sphere does not depend on the choice of coordinate system, i.e. to the directions of the $\theta=0$ axis and $\lambda=0$ plane.

The concept of spatial power spectrum should not be used for the components $X$ and $Y$ separately, because the $C_{n, n^{\prime}, m}$ terms of eqs. (2.10) and (2.11) mean that the separation into contributions from individual degrees is not possible (the values of $X$ and $Y d o$ depend on the choice of coordinate system).

James $(1968,1969)$ introduced the concept of $\left\langle X_{n}^{2}\right\rangle$ and $\left\langle Y_{n}^{2}\right\rangle$ when he was considering the total field of degree $n$ as coming from a single $n$-th order multipole. The values of $\left\langle X_{n}^{2}\right\rangle$ and $\left\langle Y_{n}^{2}\right\rangle$ in his table 2 are valid for each multipole separately, but he does not say explicitly that they cannot be added to give the total $\left\langle X^{2}\right\rangle$ and $\left\langle Y^{2}\right\rangle$. Similarly Nevanlinna (1987a) also calculates individual $\left\langle X_{n}^{2}\right\rangle$ and $\left\langle Y_{n}^{2}\right\rangle$ and plots them as «power spectra» in his fig. 1b. Of course, caution should be used when transferring the interpretation of the slope of the power spectra of $V$ and $\boldsymbol{B}$ in terms of the apparent source-layer depth (e.g. Meyer, $1985)$, to the slopes of $\left\langle X_{n}^{2}\right\rangle$ and $\left\langle Y_{n}^{2}\right\rangle$.

The concept of spatial power spectrum was introduced also for subperiodic functions (Haines, 1991, page 243), but again the problem of non-orthogonality was not considered.

\section{Integral and least-squares methods, and the use of $\boldsymbol{X} \sin \theta, Y \sin \theta$}

Given a knowledge of, say, $V(a, \theta, \lambda)$ over the sphere then, because of the orthogonality of the $\Psi_{n}^{m}$ we can obtain the values of the coeffi- cients $g_{n}^{m}$ in a spherical harmonic expansion of $V$ by the classical integration

$$
\begin{gathered}
g_{n}^{m}=\frac{\int_{4 \pi} V(a, \theta, \lambda) \Psi_{n}^{m}(\theta, \lambda) d \Omega}{\int_{4 \pi}\left[\Psi_{n}^{m}(\theta, \lambda)\right]^{2} d \Omega}= \\
=\frac{\int_{4 \pi} V \Psi_{n}^{m} d \Omega}{4 \pi<\left(\Psi_{n}^{m}\right)^{2}>}
\end{gathered}
$$

$V$ itself is not observable, but the same technique can be applied to any of the observables $\boldsymbol{B}, \boldsymbol{H}$ or $Z$, but not to $X$ or $Y$. For example, for $Z$ we have (Chapman and Bartels 1940; Kawasaki et al. 1989, where there is a typographical error in their eq. (2.2)):

$$
g_{n}^{m}=-\frac{2 n+1}{4 \pi(n+1)} \int_{4 \pi} Z(a, \theta, \lambda) \Psi_{n}^{m}(\theta, \lambda) d \Omega .
$$

In practice we do not know the field (component) over the whole sphere, but only at discrete points; some workers use a numerical approximation to the integration of the classical approach, but most workers prefer to use a least-squares approach. We now show how these two methods are related for a uniform distribution of data.

Consider a set of $M$ data $B_{i}$, taken at the measurement points $\boldsymbol{x}_{\boldsymbol{i}}=\left(\theta_{i}, \lambda_{i}\right)$, where $B$ is one of the field components, or all of them (i.e. all the complete vectorial information).

In the least-squares method we look for those coefficients that minimize the square of the Euclidean norm $S$ of the difference between data and model:

$$
S=\sum_{i}\left(B_{i}-\sum_{m, n} g_{n}^{m} B_{n}^{m}\left(\boldsymbol{x}_{i}\right)\right)^{2} .
$$

We need to solve a system of normal equations which has as many equations as the number of unknown coefficients; the $(n, m)-t h$ of them is (here the couple $(n, m)$ represents a single index):

$$
g_{1}^{0} \sum_{i=1}^{M} B_{1}^{0}\left(\boldsymbol{x}_{\boldsymbol{i}}\right) B_{n}^{m}\left(\boldsymbol{x}_{\boldsymbol{i}}\right)+\ldots+
$$




$$
+g_{n}^{m} \sum_{i=1}^{M} B_{n}^{m}\left(\boldsymbol{x}_{\boldsymbol{i}}\right) B_{n}^{m}\left(\boldsymbol{x}_{\boldsymbol{i}}\right)+\ldots=\sum_{i=1}^{M} B_{i} B_{n}^{m}\left(\boldsymbol{x}_{\boldsymbol{i}}\right) .
$$

The eq. (4.3) can be written in matrix notation:

$$
S=\|\boldsymbol{B}-\boldsymbol{A} \boldsymbol{g}\|,
$$

where $\boldsymbol{g}$ is a column vector of the $g_{n}^{m}, \boldsymbol{B}$ a column vector of the observations, and $\boldsymbol{A}$ is the matrix of the $B_{n}^{m}\left(\boldsymbol{x}_{\boldsymbol{i}}\right)$. The normal eqs. (4.4) are then

$$
\boldsymbol{A}^{T} \boldsymbol{A} \boldsymbol{g}=\boldsymbol{A}^{T} \boldsymbol{B}
$$

where the $(n, m),\left(n^{\prime}, m^{\prime}\right)$ element of $\boldsymbol{A}^{T} \boldsymbol{A}$ is the sum

$$
\sum_{i=1}^{M} B_{n}^{m}\left(\boldsymbol{x}_{i}\right) B_{n^{\prime}}^{m^{\prime}}\left(\boldsymbol{x}_{i}\right)
$$

In the ideal case of uniform (i.e. equal area) distribution over the sphere, the sums are essentially integrals, so

$$
\begin{gathered}
4 \pi \sum_{i=1}^{M} B_{n}^{m}\left(\boldsymbol{x}_{i}\right) B_{n^{\prime}}^{m^{\prime}}\left(\boldsymbol{x}_{i}\right) \rightarrow \\
\rightarrow \int_{4 \pi} B_{n}^{m}(\lambda, \theta) B_{n^{\prime}}^{m^{\prime}}(\lambda, \theta) d \Omega=4 \pi<B_{n}^{m} B_{n^{\prime}}^{m^{\prime}}>,
\end{gathered}
$$

where $d \Omega=4 \pi / M$.

We have seen that for each of $\boldsymbol{B}, \boldsymbol{H}$ or $Z$ the value of $<B_{n}^{m} B_{n^{\prime}}^{m^{\prime}}>$ is zero unless $B_{n}^{m}=B_{n^{\prime}}^{m^{\prime}}$. So $\boldsymbol{A}^{T} \boldsymbol{A}$ is, to our approximation, diagonal, and each $g_{n}^{m}$ can be determined separately, giving (almost) (4.2) again, for example using $B_{i}=Z_{i}$.

If instead we use $X$ or $Y$ separately, the discussion of section 2 shows that $\boldsymbol{A}^{T} \boldsymbol{A}$ is no longer diagonal, with all the resulting complications such as correlation and possible ill-conditioning.

Even for $\boldsymbol{B}, \boldsymbol{H}$ or $Z$, if the data are not uniformly spaced, orthogonality is lost, and $\boldsymbol{A}^{T} \boldsymbol{A}$ becomes non-diagonal. For example, if the data are equally spaced in latitude and longitude, to restore diagonality we need to weight the observations by $\sin \theta_{i}$, in effect allocating the appropriate $d \Omega$ in our approximation to the integral of (4.2) (Lowes, 1976; Langel, 1987).

Although using $Y$ gives a non-diagonal matrix, it is clear from (1.5) that $Y \sin \theta$ gives orthogonal functions $Y_{n}^{m} \sin \theta$, and so can be used in an integral approach; for equiangular data, this corresponds to weighting $Y$ data by $\sin ^{2} \theta$, and this is what Alldredge and Kawasaki (1981, page 511) suggested for data equally spaced along a meridian. Because of its orthogonality, $Y \sin \theta$ could be used to give a sort of power spectrum.

Similarly $X \sin \theta$ can be useful for an integral approach (Schmidt, 1889; Chapman and Bartels, 1940, page 637; Mauersberger, 1959, page 132; Kawasaki et al., 1989), but not in a direct way:

$$
\begin{gathered}
X \sin \theta=\sum_{n, m} g_{n}^{m} \cos m \lambda \sin \theta \frac{d}{d \theta} P_{n}^{m}(\cos \theta)= \\
=\sum_{n, m} g_{n}^{m} \cos m \lambda\left[A_{n}^{m} P_{n+1}^{m}-B_{n}^{m} P_{n-1}^{m}\right],
\end{gathered}
$$

where (Chapman and Bartels, 1940):

$A_{n}^{m}=\frac{\sqrt{(n+1)^{2}-m^{2}}}{2 n+1} n, \quad B_{n}^{m}=\frac{\sqrt{n^{2}-m^{2}}}{2 n+1}(n+1)$.

If we develop $X \sin \theta$ with a finite number of harmonics, i.e.:

$$
X \sin \theta=\sum_{n=0}^{N+1} \sum_{m=0}^{n} e_{n}^{m} \cos m \lambda P_{n}^{m}(\cos \theta),
$$

then we can evaluate the coefficients $g_{n}^{m}$ using the relation

$$
\begin{gathered}
A_{n}^{m} g_{n}^{m}+B_{n+2}^{m} g_{n+2}^{m}=e_{n+1}^{m} \Rightarrow \\
\frac{\sqrt{(n+1)^{2}-m^{2}}}{2 n+1} n g_{n}^{m}-\frac{\sqrt{(n+2)^{2}-m^{2}}}{2 n+5}(n+3) g_{n+2}^{m}= \\
=\frac{2 n+3}{4} \int_{4 \pi} X \sin \theta P_{n+1}^{m}(\cos \theta) d \cos \theta,
\end{gathered}
$$

which is the same expression given by Kawa- 
saki et al. (1989). This is a linear problem of the kind $e=C g$ :

$$
\begin{aligned}
& e_{m}^{m}=B_{m+1}^{m} g_{m+1}^{m} \\
& e_{m+1}^{m}=A_{m}^{m} g_{m}^{m}+B_{m+2}^{m} g_{m+2}^{m} \\
& e_{m+2}^{m}=A_{m+1}^{m} g_{m+1}^{m}+B_{m+3}^{m} g_{m+3}^{m} \\
& \ldots
\end{aligned}
$$

The first equation involves an isolated coefficient $\left(g_{m+1}^{m}\right)$ so, starting from it, we can evaluate all the terms $g_{m+1+2 h}^{m}(h=0,1,2, \ldots)$.

In theory, assuming that there are only a known finite number of harmonic terms, a solution for the remaining terms can be found by making a backward iteration (Mauersberger, 1959) starting from the last terms of the harmonic expansion (i.e. all the terms with order $n=N-1, N$, which are isolated as the coefficient $g_{n+1}^{m}$ seen before). But the truncation we use is subjective, and in practice the high degree term we first evaluate will be very uncertain, and in both cases the iteration will add increasing error, so the situation is very far from that of (4.2).

\section{Discussion}

The orthogonality of spherical harmonics is fundamental in geomagnetic field analysis. Spherical harmonics represent a complete and orthogonal set of functions that can be used to expand the magnetic potential satisfying Laplace's equation (e.g. Chapman and Bartels, 1940). A central role in the context of this paper is that their derivatives $\partial / \partial \theta$ and $(\sin \theta)^{-1} \partial / \partial \lambda$ are not orthogonal over the sphere. In Chapman and Bartels (1940, sec. 17.8) it is affirmed that any partial derivative of a spherical harmonic is still a solution of Laplace's equation, but it is more correct to say that it is true for derivatives with respect to fixed cartesian coordinates $x, y, z$ or, when using spherical coordinates, for $\partial / \partial \lambda$ (i.e. effectively $Y \sin \theta$ ), for which the expression of Laplace operator is linear. Similar confusion can also arise from works by Schmitz and Cain (1983, page 1225) and Haines (1991, page 244) where it is said that $\theta$-derivatives of Legendre functions of degree $n$ can be expressed simply in terms of Legendre functions of degrees $n+1$ and $n-1$, omitting the importance of the term $\sin \theta$ in the relation (56) of Chapman and Bartels (1940, page 622). Similarly, to give a compact expression of the spatial spectrum of secular variation, Nevanlinna (1987b; his eq. (6) at page 369) neglects integrals with terms like $\partial X / \partial \lambda \cdot \partial X / \partial \theta$, invoking a fictitious orthogonality.

However the total horizontal vector $\boldsymbol{H}=$ $(X, Y)$ is orthogonal.

Because of orthogonality, the concept of (one-dimensional) power spectrum in terms of spherical harmonic coefficients can be introduced for $V, \boldsymbol{B}, H$ and $Z$ because each harmonic degree contributes separately to the total power of the considered quantity. The shape of these spectra have been used to infer some properties of crustal and/or core contributions (e.g. Lowes, 1974; Langel and Estes, 1982). But the concept should not be extended to the $X$ and $Y$ components which are not orthogonal over the sphere.

$(X, Y) \sin \theta$ are useful when analysing only one of the two components, otherwise, the vector $\boldsymbol{H}$ being orthogonal over the sphere, it is better to analyze $X$ and $Y$ simultaneously. Similarly, using only $X$ or $Y$ individually, for example in a $(X, Z)$ or $(Y, Z)$ least-squares separation of the internal and external parts of the solar quiet daily variation, will give significant off-diagonal terms in the normal-equations matrix. The resultant ill-conditioning might contribute to the inconsistencies (e.g. Matsushita, $1983)$ between the $(X, Z)$ and $(Y, Z)$ analyses.

\section{Acknowledgements}

We are greatly in debt to B. Duka and A. Meloni for useful discussions and suggestions. Some useful papers were kindly furnished by G.P. Gregori, B.P. Singh and D.E. Winch. 


\section{REFERENCES}

AlldRedGe, L.R. and K. KaWASAKI (1981): Spherical harmonic analysis in the presence of high harmonics, J. Geomagn. Geoelectr., 33, 503-515.

ArfKen, G. (1985): Mathematical Methods for Physicists, (3rd edition, Academic Press, Inc. San Diego).

CAIN, J.C., Z. WANG, D.R. SChMITZ and J. MEYER (1989): The geomagnetic spectrum for 1989 and core-crustal separation, Geophys. J., 97, 443-447.

CHAKRABARTY, S.K. (1954): The spherical harmonic analysis of the Earth's main magnetic field, Indian J. Meteorol. Geoph., 5, S. 63/68.

Chapman, S. and J. BARTEls (1940): Geomagnetism (Oxford University Press, New York), vol 2.

HaINES, G. V. (1991): Power spectra of sub-periodic functions, Phys. Earth Planet. Inter., 65, 231-247.

JAMES, R.W. (1968): Multipole analysis. I. Theory, and geomagnetic multipoles 1965.0, Aust. J. Phys., 21, 455-464.

JAMES, R.W. (1969): Multipole Analysis. II. Geomagnetic secular variation, Aust. J. Phys., 22, 481-495.

JEFFRIES, H. (1942): The stress differences in the Earth's shell, Mon. Not. R. Astron. Soc., Geophys. Suppl., 5, 71-89.

KAULA, W.M. (1967): Theory of statistical analysis of data distributed over a sphere, Rev. Geophys., 5 (1), 83-107.

KaWASAKI, K., S. Matsushita and J.C. CAIN (1989): Least squares and integral methods for the spherical harmonic analysis of the Sq-Field, Pure Appl. Geophys., 131, 357-370.

LANGEL, R.A. (1987): The main field, in Geomagnetism, edited by J.A. JACOBS (I ed. Academic Press, London), vol. 1, 249-512.

LANGEL, R.A. and R.H. Estes (1982): A geomagnetic field spectrum, Geophys. Res. Lett., 9, 250-253.

LOWES, F.J. (1966): Mean-square values on sphere of spherical harmonic vector fields, J. Geophys. Res., 71 (8), 2179.

LowES, F.J. (1974): Spatial power spectrum of the main geomagnetic field, and extrapolation to the core, Geophys. J.R. Astron. Soc., 36, 717-730.

LOWES, F.J. (1975): Vector errors in spherical harmonic analysis of scalar data, Geophys. J.R. Astron. Soc., 42, 637-651.

LowES, F.J. (1976): The effect of a field of external origin on spherical harmonic analysis using only internal coefficients, J. Geomagn. Geoelectr., 28, 515-516.
LuCKE, O. (1957): Über Mittelwerte von Energiedichten der Kraftfelder, Wiss. Z. der Päd. Hochschule Postdam, Jahrgang 3, Heft 1, 39-46.

LuCKE, O. (1959): Analyse der Veränderingen des erdmagnetischen Hauptfeldes, in Geomagnetismus und Aeronomie. band III (Veb Deutscher Verlag der Wissenschaften, Berlin), 229-230.

MatsushitA, S. (1983): Cautions for the estimation of the equivalent current systems from geomagnetic data, Planet. Space Sci., 31 (10), 1199-1208.

Mauersberger, P. (1956): Das Mittel der Energiedichte des geomagnetischen Hauptfeldes an der Erdoberfläche und seine säkulare Änderung, Gerlands Beitr. Geophys., 65, 207-215.

MAUERSBERGER, P. (1959): Mathematische beschreibung und statische untersuchung des hauptfeldes und der säkularvariation, in Geomagnetismus und Aeronomie. Band III (Veb Deutscher Verlag der Wissenschaften, Berlin), 128-134.

MCLEOD, M.G. and JR.P.J. COLEMAN (1980): Spatial power spectra of the crustal geomagnetic field and core geomagnetic field, Phys. Earth Planet. Inter., 23, P5P19.

Meyer, J. (1985): Secular variation of the magnetic mean energy density at the source-layer depth, Phys. Earth Planet. Inter., 39, 288-292.

NevanlinNA, H. (1987a): Notes on global mean-square values of the geomagnetic field and secular variation, J. Geomagn. Geoelectr., 39, 165-174.

NevanlinNa, H. (1987b): On the drifting parts in the spatial power spectrum of the geomagnetic secular variation, J. Geomagn. Geoelectr., 39, 367-376.

SCHMIDT, A. (1889): Mathematische Entwicklungen zur allgemeinen Theorie des Erdmagnetismus (Archiv Deutsch. Seewarte, Hamburg), XII, n. 3.

SCHMIDT, A. (1935): Tafeln der normierten Kugelfunktionen, sowie Formeln zur entwicklung (Gotha, Verlag Engelhardt-Reyer).

SCHMITZ, D.R. and J.C. CAIN (1983): Geomagnetic spherical harmonic analysis. 1. Techniques, J. Geophys. Res., 88 (B2), 1222-1228.

STRATTON, J.A. (1941): Electromagnetic Theory (McGraw Hill, New York).

(received August 23, 1994; accepted March 28, 1995) 